\title{
The Third Hand
}

\section{Sudhakar.T, Jessie Christilda.J, Sindhuja Mary.S, Sindu Divakaran, Hemalatha RJ}

\begin{abstract}
One of the modern emerging trends in automation is robotics. Robots have been increasingly used in various industrial and non-industrial environment. Most of the robots are used for assembling and manufacturing units in automobile industries. However, in recent times robots have been replacing humans in hazardous environments in order to protect humans from exposure to dangerous elements. Thus, acting as a helping hand for humans in unviable environments. Our project is to build such a helper robot which can be used by humans to direct the robot to do his/her work in such places. For example, it can be used in pharmaceutical and biochemical industries for experiments involving volatile chemical substances. Another essential use for this type of robot is in the surveillance and maintenance work in nuclear reactors and mining industries. Thus, the robot protects human, increases manpower and work efficiency.
\end{abstract}

Keywords-8051, Arduino, Robot arm, Pot control.

\section{INTRODUCTION}

This project focuses on the use of robots as a proxy in place of humans working in hazardous environments like pathological laboratories, chemical plants, heavy work industrial plants and even nuclear power plants. The robot is guided by the human to complete the work with efficiency and thus protects the overall health of the worker without exposure to toxins and industrial hazards or wastes.

Among the many justifications for using robotics, the most important is to shield people from working in dangerous environments and from handling hazardous materials. From dealing with chemicals that are explosive to handling radioactive substances, robots are routinely used to perform tasks that would kill or maim people. Robots are ideal for use in hazardous environments by removing people from direct exposure to unfriendly conditions such as materials that are radioactive or highly explosive.

Manuscript published on November 30, 2019

* Correspondence Author

Sudhakar.T*, Assistant Professor-Department of Biomedical Engineering, Sathyabama Institute of Science \& Technology, Chennai, India.Email: tsr.182001@yahoo.co.in

Jessie Christilda.J, Student -Department of Biomedical Engineering, Sathyabama Institute of Science \& Technology, Chennai, India.

Sindhuja Mary.S, Student -Department of Biomedical Engineering, Sathyabama Institute of Science \& Technology, Chennai, India.

Sindu Divakaran, Assistant Professor Department of Biomedical Engineering, Sathyabama Institute of Science \& Technology , Chennai, India.

Hemalatha.R.J, Head \& Assistant Professor -Department of Biomedical Engineering,Vels Institute of Science, Technology \& Advanced Studies, Chennai, India. Email hodbiomedical.se@velsuniv.ac.in

(C) The Authors. Published by Blue Eyes Intelligence Engineering and Sciences Publication (BEIESP). This is an open access article under the CC-BY-NC-ND license http://creativecommons.org/licenses/by-nc-nd/4.0/
[1] Ajayi Ayodele, et.al.,developed a microcontroller based robotic arm. They used 8051 microcontroller to control the robot operations. A sample robot which can grab, and release things is built for demonstration. The method of interfacing the robotic arm stepper motor with 8051 is also explained.

[2] Abdellatif Bab et.al. controlled robotic arm with the help of arduino. The arm has the ability to move in 4 axis directions with 5 servo motors. For control they used androidapplication via Bluetooth module connected to Arduino Nano microcontroller.

[3] Chitturi Venkatratnam and Wong Sai Kit developed a pick and place robot arm using raspberry pi 2 . They designed the arm in such a way to help the user to pick the items using an extendable arm. Raspberry pi 2 controls the overall design with 5 DOF servo motor arms.

[4] Goldykatal et.al. developed a project on design and operation of synchronized robotic arm. In this paper the robotic arm is designed, synchronized with the working arm and would prefer the task of the working arm does. The programming is done on ATMEGA-8 microcontroller using arduino programming. The potentiometers are used to detect the angle of rotation and the signals sent to microcontroller.

[5] Mohammad Javed Ansari et.al. developed a microcontroller-based robot arm by giving input gestures. The system senses the movement of users arm and robotic arm replicates the give input gesture. The gesture is sensed by several potentiometers which is embedded onto a glove.

[6] Ankur Bhargava and Anjani Kumar developed an arduino based robot arm. They designed a robotic arm with 5 DOF. It is controlled by an Arduino Uno microcontroller which accepts input signals from a user by means of a potentiometer. The Arduino has been programmed to provide rotation to each servo motor corresponding to the amount of rotation of the potentiometer shaft.

[7] Ron Oommen Thomas and Prof.K.Rajasekaran developed a robotic arm using raspberry pi. They used internet to control the robotic arm from a remote end. The control signals to robotic arm were provided by the raspberry pi and are the causes for arm movements.

[8] Chein-weiChen developed an EMG based robot arm to use robot without touching in factories. [9-11] Instead of using myoelectric signals we use a wired pot joystick for the same concept of using the robot arm without human touch.

Ferdinando Cannella proposed a 4 DOF gripper which is graduated from the top to easily grip objects including cylinder like structure. We use a modified claw like gripper to provide good pick and place function.

\section{METHODOLOGY}

The robot will be a movable robot operated on wheels powered by a geared dc motor controlled by a microcontroller.

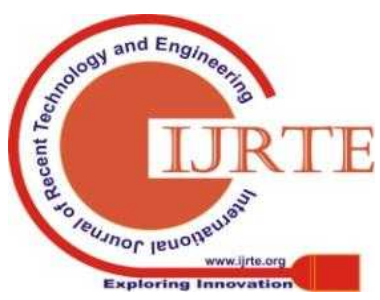




\section{The Third Hand}

A robotic manipulator in form of an arm will be attached to the base. The robot arm will be controlled by Arduino processor along with a potentiometric joystick. The end effector of the robot hand is graduated with claw like grippers. The work volume of the robot depends upon the wire length. The robot has 4 degrees of freedom excluding end effector movement.

\section{Hardware components}

Several hardware components have been used to design the device and to make it function. The main components are

- 8051 microcontroller

- Servo motor

- DC motor

- 10k pot,

- $\quad$ SMPS (switched mode power supply)

- Arduino

- other minor components.

8051 Microcontroller: It is the processor used for the mobility of the robot. Here 8051 microcontroller is used with AT89S52 IC.

Servo motor: Its task is to move to and maintain itself at a angular position. Most servos are between 0 to 180 degrees. It's easy to control servos. It's a mechanical as well as electronic module. It has a small potentiometer in the box itself to take feedback.

Geared DC motor: The specifications of the motor is $12 \mathrm{~V}$ with $60 \mathrm{rpm}$ speed without load. It has high speed control and high starting torque. And has quick starting, stopping, reversing and acceleration.

$10 \mathrm{k}$ Pot is a three-termina lresistor. Here we use it as a position transducer to define the various angles for servo motor thereby defining arm position.

SMPS (Switch Mode Power Supply): The servo motors need an external power supply which cannot be powered by the Arduino board. Hence, we use 5V 10A SMPS for linear regulated supply.

Arduino Uno: is used for arm positioning and control along with servo motor. The Arduino board actually is a specially designed circuit board for programming and prototyping with Atmel microcontrollers. The nice thing about the Arduino board is that it is relatively cheap, plugs straight into a computer's USB port, and it is dead-simple to setup and use (compared to other development boards).

\section{Software components}

- Keil Software

- $\quad$ PROGisp

The keil software programs to compile the $\mathrm{C}$ code assemble your assembly source files, link and locate object modules and libraries, create hex files, anddebug your target program. Hex file is a format used to store machine language code in hexadecimal form. It is widely used format to store programs to be transferred to microcontrollers, ROM and EEPROM. The compilers convert the programs written in assembly, $\mathrm{C}$ etc. into corresponding hex files, which are dumped into the controllers using burners/programmers. The microcontroller understands machine language consisting of zeroes and ones. It's difficult rather practically impossible for humans to write codes in zeros and ones. Hence, we use some high-level languages like $\mathrm{C}, \mathrm{C}++$, Java, etc. And later a compiler is used to convert these codes into machine language which are stored in a hex file format. A hex file is a text file with the extension. hex.

PROGisp: To transfer the Hex file to controller we require some software to access USBASP. USB asp is a USB in-circuit programmer for Atmel AVR controllers. It simply consists of an ATMega8 and a couple of passive components. The programmer uses a firmware-only USB driver, no special USB controller is needed, and that makes it low cost USB programmer. PROGISP is the software used to load the HEX file on to the target chip.

Wheel control for robot using 8051

The SMPS is connected to the AC mains and turned on. It powers the 8051, Arduino Uno and the robot arm. The SMPS serves as the primary power supply.

The body of the robot primarily made of an acrylic chassis AkshayHiwase etal., [10] with four wheels with the microcontroller, Arduino, SMPS and robot arm fixed onto the chassis.

The wheels are powered separately by a $12 \mathrm{~V}$ battery through two motor drivers for the robot to move in four different directions Vibhor Gupta.

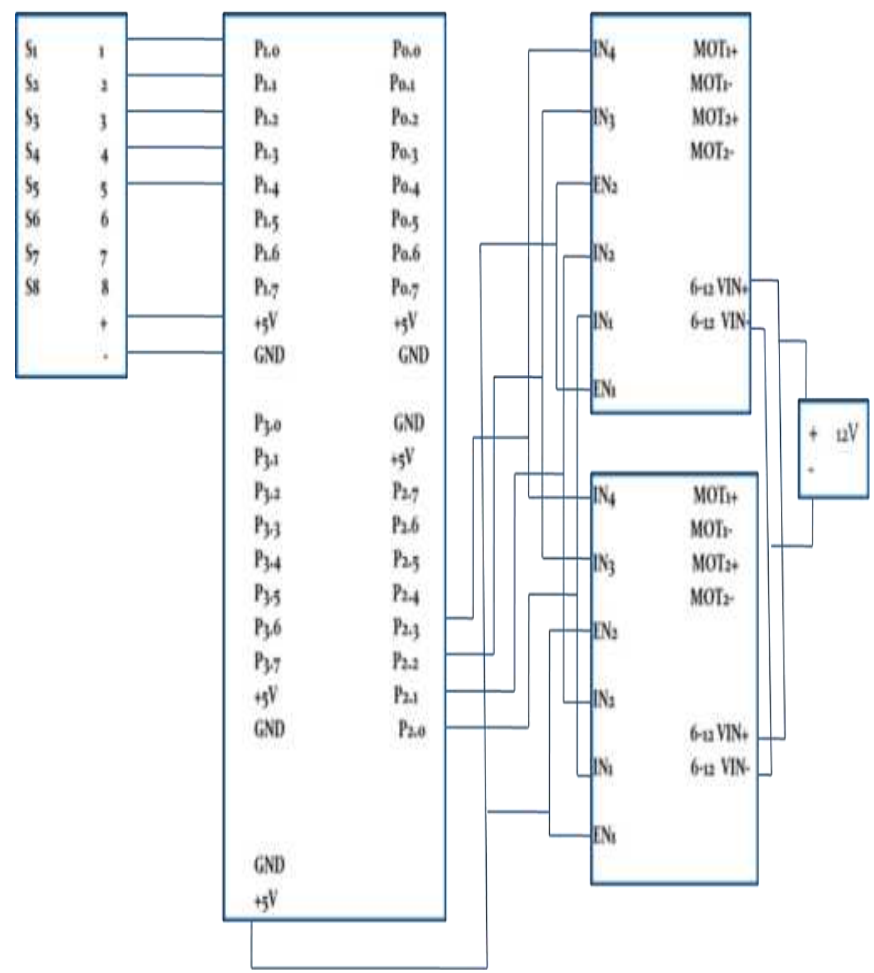

Figure 1. Pin diagram of 8051 for wheel control

The four wheels are connected to the two-motor driver and the EN pins, the IN and OP pins of the two motor drivers

were connected in tandem and was given to the $+5 \mathrm{~V}$ and port 3 pins of the microcontroller board respectively.

The wheels powered by the DC motor are controlled by the microcontroller by triggering the switch board which is connected to the ports 1.1,1.2,1.3,1.4,1.5, Vin and Gnd pins of the 8051 board. 
The 5 port pins correspond to forward, backward, left, right and stop motions of the robot wheels respectively. Thus, enabling robot movement.

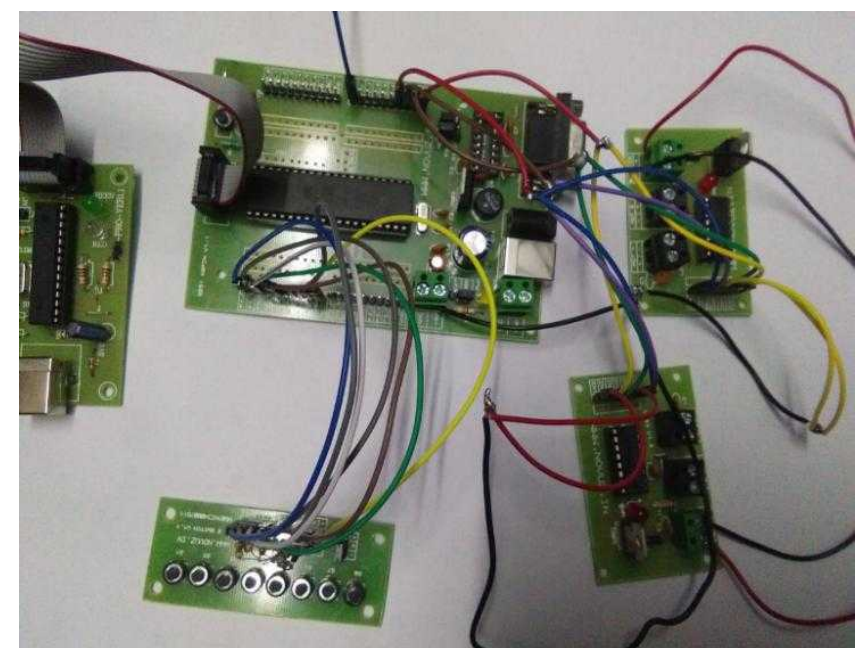

Figure 2. PCB connection for DC motor wheel control

The program for movement of DC motor control for the wheel movement was written in $\mathrm{C}$ using Keil IDE compiler and the hex file was burned onto to the IC pin using PROGisp software.

\section{Robot arm control using arduino}

The arm has 4 degrees of freedom and is powered by 5 servo motors. The servo motors signal pins are connected to PWM pins 3,5,7,9,10 respectively. The +ve and Gnd pins are connected to the SMPS for power supply.

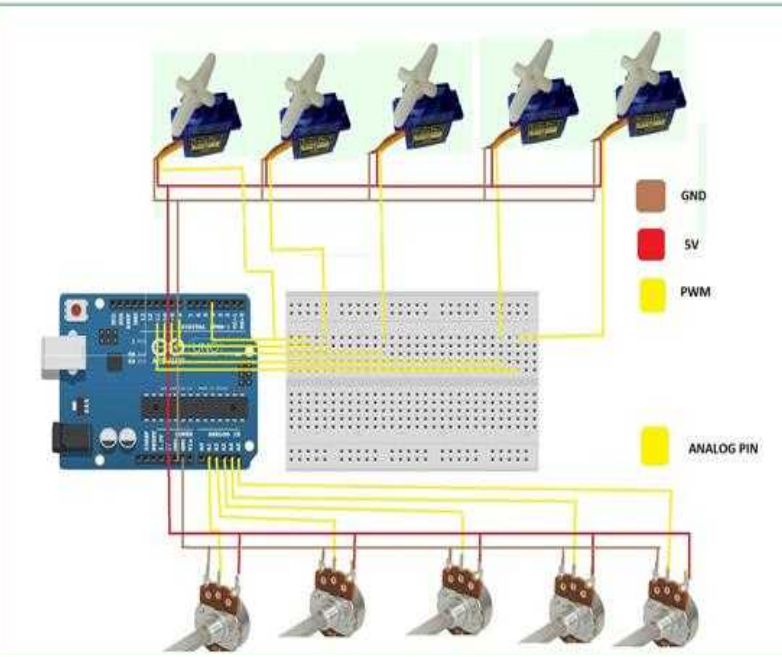

Figure 3. Arduino connected to pot and servo

Five pots are used to define the position of the arm and end effector corresponding to the rotation of the knob through 0 to $180^{\circ}$. The +ve and Gnd of the pots are soldered together and given to $+5 \mathrm{v}$ and Gnd pin in the Arduino board. The signal pins are connected to the analog pins A0, A1, A2, A3 and A4 of the Arduino.

The signal to move the servo to move the arm at certain angles are read by the Arduino from the pot signal pin and relayed to the servo via the PWM pins. Thus, enabling arm movement with respect to the pot position.

The pots are implemented in a joystick control and can be used to control arm movement at a distance. results and discussion

This robot is designed to help humans working in hazardous industrial conditions so that they might be shielded from toxic and industrial wastes. Figure 4 shows the hardware of the robot body and arm which comprises of a microcontroller, Arduino, arm, SMPS along with wheels.

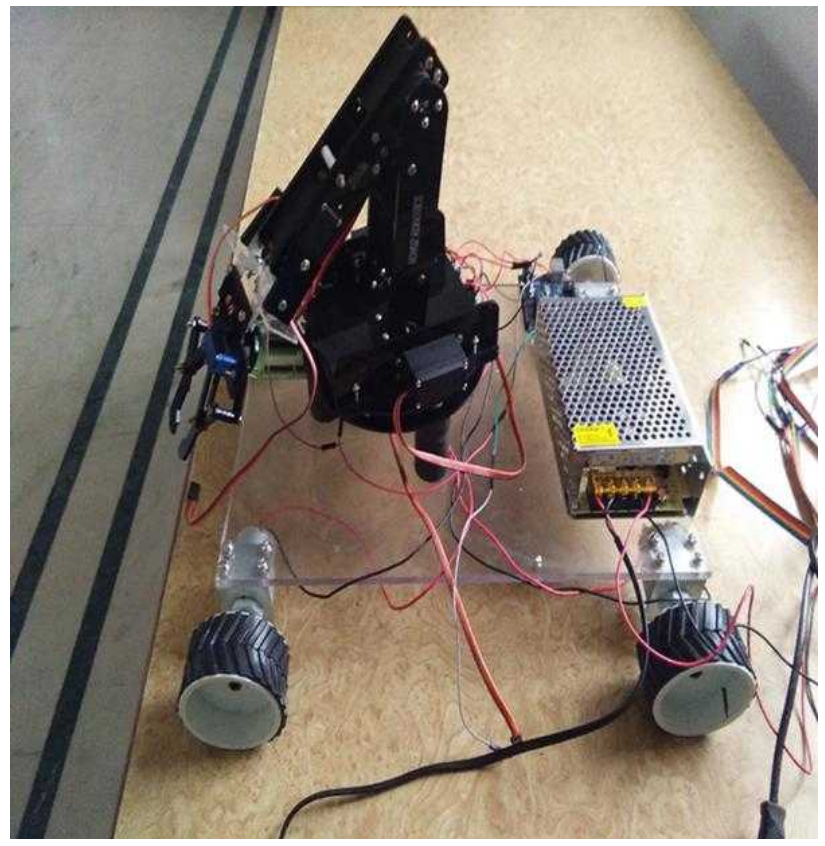

Figure 4. The third hand robot

The robot provides ample arm movement and wrist movement enabling 4 degrees of motion.

The result of the device is tested by running it and is found that the arm moves smoothly when the pot is moved along with the joystick.

The wheel allows for the 4 movements that is forward, backward, left and right movements along with stop function.

The pick and place action functions well.

Potentiometer implemented in joystick is used for the arm movement. The arm position is accurately mimicked by the motor in the arm from the output of the potentiometer.

Stable minor vibrations are found in the arm due to human error the functioning of the robot arm has found to be $95 \%$ efficient could also be due to coding discrepancies or human error or loose connection in the hardware the switch and the pot along with joystick ribbon wire to allow a wide range of movement.

The limitation was the slight vibration with noise in servo due to power discrepancies in the SMPS due to wired connection which could be corrected in the future by using wireless connection by using IoT.

\section{CONCLUSION}

Hence, it is concluded that The Third Hand is useful for the society as well as industrial application. It works successfully at the time of demonstration. In the future, it will work on wireless technology and IOT with good accuracy and precision.

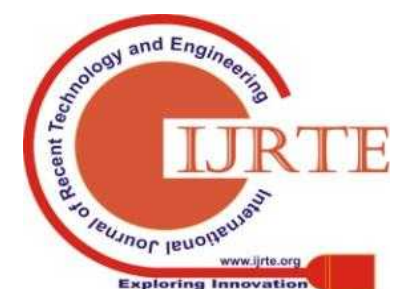




\section{REFERENCES}

1. Ajayi Ayodele et al : Proceedings of Computer science and IT education Conference : 2007 : 549-557.

2. Abdellatif Baba et al: Robotarmcontrolwitharduino: 2017 : 1-42

3. ChitturiVenkatratnam, Wong Sai Kit : IEEE Robotics and Manufacturing Automation (ROMA), 2016: 1-4.

4. GoldyKatal, Saahil Gupta, ShitijKakkar : International Journal of Engineering Research and Technology (IJRET) : 2013: 297-301.

5. Ahsanul Hoque Md., Ali Amir, Mohammad Javad Ansari: ICEEICT: 2014: 1-5.

6. Anjani Kumar, Ankur Bhargava : International Conference on Electronics Communication and Aerospace Technology (ICECA) : 2017: pp. 376-380

7. Rajasekaran.K, Ron Oommen Thomas: International Journal of Computer Application (IJCA) : 2014: pp. 29-32.

8. Amit Eytan, Aldo Bottero et al : IEEE/SICE International symposium on System Integration (SII) : 2013: 443-448.

9. Chein-Wei Chen et al : IEEE Green Technology and Sustainable Development (GTSD) : volume 1, 2016: 22-23

10. AkshayHiwase : International Journal of Advance Research and Innovative Ideas in Education (IJARIIE): Volume 4 : 2018 : 12071210 .

11. Vibhor Gupta : International Conference on Advances in Computing and Communictions (ICACC) : Volume 5 : 2010: 441-444.

\section{AUTHORS PROFILE}

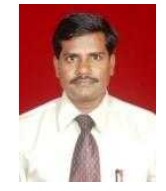

Mr.T.Sudhakar, He started his carrer as a Lecturer in the year 2001 in the Department of Microbiology, Jaya College of Arts and Science, Thiruninravur Chennai. He was working in the field of Medical Microbiology. He has renowed his research area in the field of Medical and Clinical Studies. He joined in the Department of Biomedical Engineering, Sathyabama University as a Assistant Professor in 2008.

Ms.JessieChristilda. J - is currently pursuing final year B.E. Biomedical Engineering in Sathyabama Institute of Science \& Technology- Chennai.

Ms.Sindhuja Mary.S - is currently pursuing final year B.E. Biomedical Engineering in Sathyabama Institute of Science \& Technology- Chennai

Ms.Sindu Divakaran - is currently working as Assistant Professor in . Biomedical Engineering Department in Sathyabama Institute of Science \& Technology- Chennai

Mrs.R.J.Hermalatha, is currently working as Head \& Assistant Professor in Biomedical Engineering Department in Vels Institute of Science Technology \& Advanced Studies- Chennai. She has 14 years of Teaching and research Experience. 